\title{
Comparison of a Recombinant-antigen Enzyme Immunoassay with Treponema pallidum Hemagglutination Test for Serological Confirmation of Syphilis
}

\author{
Islay Rodríguez ${ }^{+}$, Elvio LÁlvarez,Carmen Fernández, Alina Miranda
}

\author{
Departamento de Bacteriología-Micología, Instituto "Pedro Kourí", Casilla Postal 601, Marianao 13, Ciudad de La Habana, Cuba
}

A recombinant-antigen enzyme immunoassay (EIA), BioSCREEN ${ }^{T M}$ anti-Treponema pallidum, was compared favorably with the T. pallidum hemagglutination test, in the detection of specific antibodies in different groups of sera from patients with primary $(n=38)$, secondary $(n=10)$, early latent $(n=28)$ and congenital syphilis $(n=2)$, patients with leptospirosis $(n=8)$, infectious mononucleosis $(n=7)$, hepatitis $(n=9)$, diabetes mellitus $(n=11)$, rheumatoid arthritis $(n=13)$, leprosy $(n=11)$, tuberculosis $(n=9)$, HIV/Aids $(n=12)$, systemic lupus erythematosus $(n=4)$, rheumatic fever $(n=3)$, old-persons $(n=9)$, pregnant women $(n=29)$ and blood donors $(n=164)$. The coincidence between them was $95.1 \%$. The sensitivity and specificity of the EIA were $93.3 \%$ and $95.5 \%$, respectively. Fifteen serum specimens belonging to old-persons, pregnant women, blood donors, and patients with human leptospirosis, hepatitis, diabetes mellitus, tuberculosis and rheumatic fever gave false-positive results by Venereal Disease Research Laboratory and/or Rapid Plasma Reagin. The EIA can be used as alternative method for the serological confirmation of syphilis.

Key words: syphilis - Treponema pallidum hemagglutination - enzyme immunoassay

Syphilis is one of the most prevalent sexually transmitted infections worldwide. Its control and surveillance require careful screening tests by reliable methods. Serological diagnosis of syphilis should combine a nontreponemal test such as the Venereal Disease Research Laboratory (VDRL) test or the Rapid Plasma Reagin (RPR) test with a treponemal test such as the Treponema pallidum hemagglutination test (TPHA). Positive results by one or both tests could be confirmed by the Fluorescent Treponema Antibody Absorption (FTA-ABS) test. It is a very sensitive test at all stages of syphilis, but reading of fluorescence is subjective and sometimes difficult (Larsen et al. 1995).

There is a need for a reliable, specific, rapid, and automated test for the screening and confirmation of syphilis. Enzyme immunoassays (EIAs) for the detection of $T$. pallidum antibodies have been developed to achieve this aim (Ebel et al. 1998). The use of recombinant T. pallidum antigens instead of a poorly defined mixture of antigens from wild-type T. pallidum which may be mixed with rabbit testicular components, has the potential for improving the specificities of serological tests (Fujimura et al. 1997, Young et al. 1998).

A micro-EIA using as antigen the protein Tmp-A, obtained by a recombinant way at high levels in Escherichia coli (BioSCREEN ${ }^{\mathrm{TM}}$ anti-T. pallidum, Heber Biotec

\footnotetext{
${ }^{+}$Corresponding author. Fax: +537-204 6051.

E-mail: islay@ipk.sld.cu

Received 23 April 2001

Accepted 12 December 2001
}

S.A., Cuba) was compared with the TPHA (OXOID, Diagnosis Reagents, England) test in the detection of specific antibodies. The tests were performed according to the manufacturer's instructions.

Sera from patients with primary, secondary, early latent and congenital syphilis were used at the study. Sera from patients with human leptospirosis, infectious mononucleosis, hepatitis, diabetes mellitus, rheumatoid arthritis, leprosy, tuberculosis, HIV/Aids, systemic lupus erythematosus, rheumatic fever, old-persons, pregnant women and blood donors were also used. Patients with infectious or noninfectious diseases were confirmed by their clinical characteristics and laboratory tests.

Table I shows the results of the different serological tests applied to the studied sera groups. The percentage of agreement between the treponemal tests (TPHA and EIA anti-Tmp A) was 95.1\% (349/367), they differed only in the $4.9 \%(18 / 367)$ of the studied sera. The main differences were observed in the groups of other diseases, in which the nontreponemal serology was nonreactive.

The obtained data were analyzed according to the EPIDAT program (version 2) to determine the qualitative parameters of the EIA anti-TmpA, and the results are showed in the Table II.

The EIA showed sensitivity and specificity comparable with the TPHA test's (96.1\% and $100 \%$, respectively).

Statistical differences were not found in the results of the tests. Moreover, we found a patient having any of the following diseases or conditions: hepatitis, HIV/Aids, oldperson as five blood donors whose sera did not react in the nontreponemal tests, with EIA anti-TmpA positive and TPHA negative, suggesting that these persons may have previously had syphilis that was treated, with no detectable TPHA titers, or false-positive reactions. 
TABLE I

Results of the different serological tests applied to the studied sera groups

\begin{tabular}{lcccc}
\hline Group of sera in study & Number of specimens & $\begin{array}{c}\text { VDRL and/or RPR } \\
\text { positive }\end{array}$ & $\begin{array}{c}\text { TPHA positive } \\
\text { positive }\end{array}$ & $\begin{array}{c}\text { EIA } \\
\text { anti-TmpA }\end{array}$ \\
\hline Primary syphilis & 38 & 37 & 35 & 10 \\
Secondary syphilis & 10 & 10 & 28 & 10 \\
Early latent syphilis & 28 & 28 & 2 & 24 \\
Congenital syphilis & 2 & 2 & 0 & 2 \\
Human leptospirosis & 8 & 2 & 0 & 0 \\
Infectious mononucleosis & 7 & 1 & 0 & 1 \\
Hepatitis & 9 & 1 & 0 & 0 \\
Diabetes mellitus & 11 & 4 & 0 & 0 \\
Rheumatoid arthritis & 13 & 0 & 0 & 0 \\
Leprosy & 11 & 0 & 0 & 0 \\
Tuberculosis & 9 & 2 & 0 & 0 \\
HIV/Aids & 12 & 0 & 0 & 0 \\
Systemic lupus erythematosus & 4 & 0 & 0 & 1 \\
Rheumatic fever & 3 & 1 & 0 & 0 \\
Old-persons & 9 & 2 & 0 & 0 \\
Pregnant women & 29 & 3 & 75 & 83 \\
Blood donors & 164 & 2 & 95 & 0 \\
Total & 367 & & 0 & 0 \\
\hline
\end{tabular}

VDRL: Venereal Disease Research Laboratory; RPR: Rapid Plasma Reagin; TPHA: Treponema pallidum Hemagglutination; EIA anti-TmpA: Enzyme Immunoassay anti-protein TmpA of $T$. pallidum

TABLE II

Qualitative parameters of the Enzyme Immunoassay antiprotein TmpA of Treponema pallidum

\begin{tabular}{lcc}
\hline Parameter & Value $(\%)$ & Range (CI 95\%)(\%) \\
\hline Sensitivity & 93.3 & $84.5 ; 97.5$ \\
Specificity & 95.5 & $92.3 ; 97.5$ \\
Positive predictive value & 84.3 & $74.3 ; 91.1$ \\
Negative predictive value & 98.2 & $95.7 ; 99.4$ \\
Concordance & 95.1 & $92.2 ; 96.3$ \\
\hline
\end{tabular}

$\mathrm{J}$ of Youden $=0.889(0.827 ; 0.950)$

Kappa $(\mathrm{K})$ value $=0.855(0.789 ; 0.919)$

Standard error of $\mathrm{K}=0.03377(\mathrm{p}<0.01)$

Fifteen sera with reactive nontreponemal tests were identified as false-positive results by these assays, while others three sera resulted positive by the EIA anti-TmpA but negative by TPHA. A possible cause of this discrepancy could be there were some impurities or epitopes from $E$. coli at the recombinant antigen as a result of its production, which could promote nonspecific cross-reactions. We thought in this possibility because TPHA showed a $100 \%$ of specificity in this group of sera. In these cases the FTA-ABS test (gold standard treponemal test) could be necessary to resolve the discrepancies, though falsenegative results with this assay have been found by others authors (Sordillo et al. 1998). False-positive results were detected in sera from old-persons, pregnant women, blood donors and patients with human leptospirosis, hepatitis, diabetes, tuberculosis and rheumatic fever. The VDRL and/or RPR titres were inferior to 1:8. These results are consistent with literature (Koskenvuo et al. 1989, Larsen et al. 1995, Nandwani \& Evans 1995). Sera from patients with rheumatoid arthritis and systemic lupus erythematosus did not cause false-positive results in our study. In some cases, the results are difficult to assess because syphilis may actually coexist with these other infections.

The EIA anti-TmpA did not detect specific antibodies in three sera from patients with early primary syphilis. Treponemal tests vary in their reactivity in early primary syphilis; the varied sensitivities of these tests in primary syphilis are related to the time of serum collection after lesion development. In these cases the diagnosis is mainly based in clinical manifestations and epidemiological characteristics of the patient, plus a direct assay of the specimen from the lesion by dark field microscopy (Larsen et al. 1995).

The EIA anti-TmpA is easy to use as an automated test and demonstrates its usefulness for confirmation of positive results from nontreponemal tests. The main advantages of this test are the capacity to process large numbers of samples and the automated readout. No longer is the reading of the test subjective, as for the TPHA test, but a spectrophotometric determination is made and an objective reading is provided as a printout. The EIA antiTmpA represents an alternative methodology for the serological confirmation of syphilis.

\section{REFERENCES}

Ebel A, Bachelart L, Alonso JM 1998. Evaluation of a new competitive immunoassay (BioELISA Syphilis) for screening for Treponema pallidum antibodies at various stages of syphilis. J Clin Microbiol 36: 358-361.

Fujimura K, Ise N, Ueno E, Hori T, Fujii N, Okada M 1997. Reactivity of recombinant Treponema pallidum (r-Tp) antigens with anti Tp-antibodies in human syphilitic sera evaluated by ELISA. J Clin Lab Anal 11: 315-322.

Koskenvuo M, Leikola J, Palosuo T, Vaarala O, Aho K 1989. 
False-positive seroreactions for syphilis as a harbinger of disease revisited. Clin Exp Rheum 7: 75-78.

Larsen SA, Steiner BM, Rudolph AH 1995. Laboratory diagnosis and interpretation of tests for syphilis. Clin Microbiol Rev 8: 1-21.

Nandwani R, Evans DTP 1995. Are you sure it's syphilis? A review of false positive serology. Int J STD AIDS 6: 241-
248.

Sordillo EM, Hoehl B, Belch J 1998. False-negative fluorescent treponemal tests and confirmation of syphilis infection. $J$ Infect Dis 178: 294-295.

Young H, Moyes A, Seagar L, McMillan A 1998. Novel recombinant-antigen enzyme immunoassay for serological diagnosis of syphilis. J Clin Microbiol 36: 913-917. 
350 EIA anti-TmpA and TPHA • Islay Rodríguez et al. 